\title{
Plastic collapse load analysis for circumferential cracked cylinder under pure torsion
}

\author{
Sutham Arun ${ }^{1 *}$ and Thongchai Fongsamootr ${ }^{2}$ \\ ${ }^{1}$ School of Engineering, University of Phayao, Phayao, Thailand \\ ${ }^{2}$ Department of Mechanical Engineering, Chaing Mai University, Chaing Mai, Thailand
}

\begin{abstract}
This paper aims to analyze the plastic collapse moment of circumferential cracked cylinder under pure torsion using the NSC approach and 3D FE model. The material considered in this work is assumed to be elastic-perfectly plastic. The influences of geometric parameters of crack and cylinder, such as $R_{m} / t, a / t$ and $\theta / \pi$ on solution of plastic collapse load are also investigated. The analysis shows that for the case of $a / t<0.75$, the values of limit torsion moment can be estimated by NSC analysis which provides conservative results. However, for the case of deeper crack, $a / t \geq 0.75$, the limit load solution predicted by NSC approach may not be safe, because the distribution of stress at yielding state does not correspond to the NSC assumption. Therefore, the approximated solution of collapse torsion moment for the case of deeper crack with $a / t \geq 0.75$ is proposed based on FE analysis.
\end{abstract}

\section{Introduction}

The cylinder is one of the most commonly used components in a wide range of industries. During operation, it may experience various load types which lead to in-service cracking. When cracks are discovered, the analysis of plastic limit load is often performed. This is because the plastic limit load is the important key in structural integrity assessment of the cylinder.

At present, analytical and finite element (FE) solutions of limit load have been well documented for circumferential cracked cylinders [1-4]. These solutions are available for Mode I type of loading of internal pressure, axial tension and bending. However, in addition to pressure, tension and bending, the cylinder is also subjected to torsion due to spatial arrangements. Therefore, the failure assessment method without taking the effect of torsion into account may be incomplete and may affect the operational safety.

Recently, there have been few studied about limit load solution for cracked cylinder subjected to combined loading which include the effect of torsion into their works. For example, Yinsheng Li et al. [5] studied the effect of torsion moment on the limit load solution of cylinder with circumferential part-through thickness crack using FE analysis. They proposed the method for estimating the collapse load for cracked cylinder with the ratios of crack depth to wall thickness ranging between 0.5 and 0.75 . Kunio Hasegawa et al. [6] extended the work proposed by Yinsheng Li et al. They found that when the value of torsion moment is less than $20 \%$ of yield stress, the plastic collapse moment for circumferential cracked cylinders under combined bending and torsion moment can be estimated by the pure bending moment.
As mentioned above, the details about effects of torsion on the limit load solution is still inadequate. Therefore, the main aim of this paper is to provide the limit load solution for circumferential cracked cylinder subjected to pure torsion. The method used in the paper is based on NSC and FE analysis. The information obtained from this work can be used to support the structural integrity assessment of cracked cylinder.

\section{Limit Load Solution Based on NSC Approach}

Figure 1 shows the geometry of a cracked cylinder considered in this work. The outer diameter, mean radius and wall-thickness are denoted by $D_{0}, R_{m}$ and $t$, respectively. The crack is assumed to be symmetric with respect to the $y$-axis where $2 \theta$ and $a(\xi)$ represent the total angle of the crack and the crack depth in a function of angular coordinate $\xi$.

Assuming that there is only torsion exists through all the cross section along the axial direction of the cylinder, there will be only shear stress, $\tau$, acting on any cross section of cylinder. According to this, the applied torsional moment, $T$, can be computed from [7];

$$
T=\int \rho(\tau d A)
$$

where $\rho$ is the radial distance from the axial of the cylinder to any small area $d A$.

According to the NSC approach, when the material has already yielded, the stresses will uniformly distributed over the net section and are equal to the value corresponding to the yielding condition [1]. From this, the

\footnotetext{
*Corresponding author: sutham.arun@gmail.com
} 
plastic collapse moment, $T_{f}$, for the cracked cylinder defined in Figure 1 can be computed based on Eq.(1) by

$$
T_{f}=\frac{2}{\sqrt{3}} t \sigma_{f} R_{m}^{2}\left[\pi-\frac{1}{t} \int_{0}^{\theta} a(\xi) d \xi\right]
$$

where $\sigma_{f}$ is the flow stress of the cylinder material.

For a constant-depth crack which $a(\xi)=a_{0}$, Eq.(2) can be reduced to

$$
\frac{T_{f}}{T_{0}}=1-\frac{a_{0}}{t} \frac{\theta}{\pi}
$$

where the ratio $T_{f} / T_{0}$ is the normalized formulation of $T_{f}$ and

$$
T_{0}=\frac{2}{\sqrt{3}} \pi R_{m}^{2} t \sigma_{f}
$$

represents the value of collapse torsion moment for uncracked cylinder. Eq.(3) clearly illustrates that the value of collapse torsional moment is directly proportional to the ligament area of the crack section.
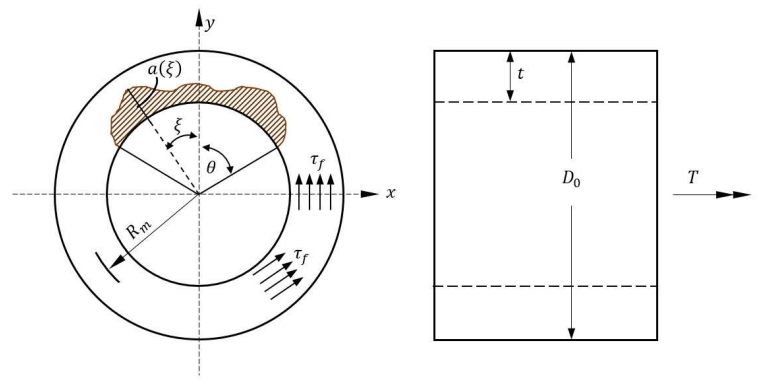

Fig. 1. Nomenclature and stress distribution of a cylinder with a circumferential crack under pure torsion.

\section{Finite element model for circumferential cracked cylinder}

In order to investigate the correctness of Eq.(3), the FE models of cylinder with circumferential crack were established in ABAQUS [8]. The continuum element with 20 nodes, reduced integration (C3D20R) was used throughout the models. To improve the accuracy of the model, partitions were created on the cracked zone in order to form a dense mesh along the crack front. During analysis, the number of element in each model was increased ranging from 15,000 elements to 38,000 elements in order to confirm that the results obtained from FE analysis are mesh-independent. Details of the model construction are given in the following sections.

\subsection{Model construction}

The FE-model of cylinder employed in this work is illustrated in Figure 2. In all cases, the outer radius of the cylinder is $300 \mathrm{~mm}$, and the length of the cylinders is set to be longer than $8 D_{0}$ in order to avoid boundary effects on the crack ligament $[5,6]$.

The circumferential crack is introduced into the middle section of the FE model. Geometric parameters of crack and cylinder are systematically varied to quantify their effects. These factors are the ratio of mean radius to wall thickness $\left(R_{m} / t\right)$, the ratio of crack depth to wall thickness $(a / t)$ and the half angle of crack $(\theta / \pi)$. The cases considered in this work are summarized in Table 1.

The material used for cylinder is a high-ductility stainless steel $[5,6]$. The Young's modulus is selected as $200 \mathrm{GPa}$ with Poisson's ratio of 0.3 . The stress-strain relationship is assumed to be elastic-perfectly plastic and the value of yield stress is equal to $3.72 \mathrm{MPa}$.

Two reference nodes are created at the center of cross section at end surfaces. The motion of these reference nodes is coupled with the motion of all nodes on each end surfaces by coupling constraints. All translation and rotation of left side reference node are prevented, and the torsional angle is applied to the right side reference node.

As the FE analysis does not directly provide the limit load, the twice-elastic-slope (TES) method is chosen to define the "plastic collapse moment". This is because TES method is suitable for elastic-perfectly plastic materials which their load deformation curves show apparent collapse behavior [9].

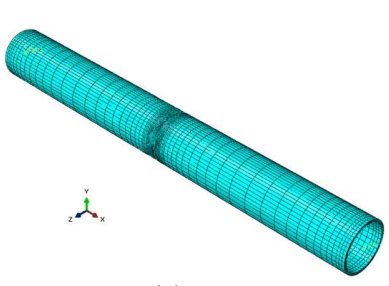

(a)

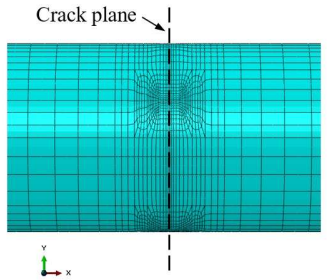

(b)
Fig. 2. (a) The specimen mesh overview and (b) mesh near the crack in longitudinal direction.

Table 1. Cases considered in the present FE analysis.

\begin{tabular}{|l|c|l|}
\hline \multicolumn{1}{|c|}{ Geometric parameter } & Symbol & \multicolumn{1}{|c|}{ Values } \\
\hline $\begin{array}{l}\text { The ratio of mean radius to } \\
\text { wall thickness }\end{array}$ & $R_{m} / t$ & $5,10,20$ \\
\hline $\begin{array}{l}\text { The ratio of crack depth to } \\
\text { wall thickness }\end{array}$ & $a / t$ & $\begin{array}{l}0.25,0.50, \\
0.75,1.00\end{array}$ \\
\hline The half angle of crack & $\theta / \pi$ & $\begin{array}{l}0.0625,0.125, \\
0.250,0.375\end{array}$ \\
\hline
\end{tabular}

\subsection{Validation of the model}

To gain confidence in the present FE analysis, the FE-model was first loaded by assuming that the cylinder has no crack. The collapse torsion moment obtained from the FE analysis is compared with the solution in Eq.(4). The results show that the present FE results agree very well with the analytical solution, Eq.(4), with the difference less than $0.1 \%$. Further confidence is gained by comparing FE results with the results computed from the equation in elastic theory. This equation is [7]

$$
\beta=\frac{L}{G J} T=C T
$$


where $\beta$ is the angle of twist, $G$ is the modulus of elasticity in shear, $L$ is the cylinder length and $J$ is the polar moment of inertia of the cross-section area. The compliance, $C$, in Eq.(5) is the ratio of torsional moment to the angle of twist.

The relationship between $T$ and $\beta$ obtained from FE analysis and Eq.(5) are plotted together in Figures 3. It can be seen that in elastic region, the relationships obtained from both analysis are in good agreement.

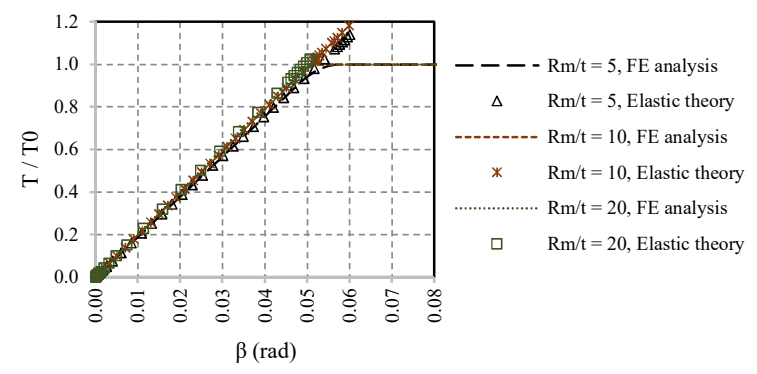

Fig. 3. The relationship between torsional moment and torsional angle (comparison between FE analysis and elastic theory).

\section{Results and discussion}

\subsection{Global behaviour}

Figure 4 presents the relationship between the normalization of applied torsional moment, $T / T_{0}$, and $\beta$ obtained from FE analysis for the cases of $R_{m} / t=5$ and $R_{m} / t=10$. It is very clear that when the cylinder has a crack, the change of crack ligament area, $a / t$, does not affect the value of $C$ in elastic region. In other words, the reduction of the ligament area in the crack section does not affect the value of $J$ in Eq.(5). This means that when material behaves elastically, the elastic theory can still be used to predict the global behavior of the circumferential cracked cylinder.

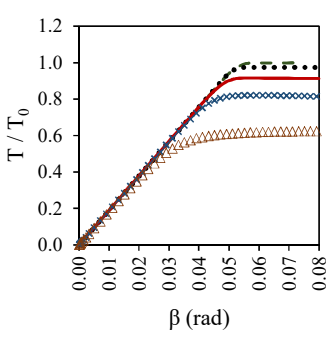

(a)

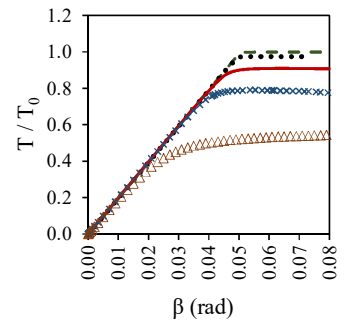

(b)
Fig. 4. Relationship between $T / T_{0}$ and torsional angle : (a) $R_{m} / t=5$ and (b) $R_{m} / t=10$.

\subsection{Effect of geometric factor of cracked cylinder on limit load}

The influences of $R_{m} / t, a / t$ and $\theta / \pi$ on the normalized limit torsional moment, $T_{f} / T_{0}$, are presented in Figures 5. It can be seen that when the value of $a / t$ is less than 0.75 , the ratio $R_{m} / t$ affects slightly, not over $10 \%$, on the change of $T_{f} / T_{0}$. However, when the value of $a / t$ is higher, the limit torsion moment changes significantly according to the value of $R_{m} / t$. This behavior is inconsistent with Eq.(3) which clearly indicates that the values of $T_{f} / T_{0}$ only depends on the ligament area of the crack section.

Figure 5 also shows that, at $a / t<0.75$, the solutions of $T_{f} / T_{0}$ obtained from the FE-analysis are slightly higher than those predicted by Eq.(3). However, when $a / t$ is higher, the values of $T_{f} / T_{0}$ provided by FE-analysis drop rapidly to lower than those predicted from Eq.(3). The reason of this behavior can be explained by the concept of equilibrium of torsion moment in Eq.(1) as follows;

Figure 6 presents the distribution of Von-Mises stress on the cracked plane at the state of yielding. It can be seen that when the cylinder has no crack, Figure $6 \mathrm{a}$, the stress acting on this cross-section area is uniform and is equal to the value of yield stress $(3.72 \mathrm{MPa})$. This corresponds to the assumption of NSC analysis. Therefore, for the case of uncracked cylinder, the ratio $T_{f} / T_{0}$ estimated by the FE-analysis is very close to the solution calculated from Eq.(3).

For the cracked cylinder with $a / t<0.75$, the stresses in the region ahead the crack front become singular after subjecting to torsional moment, as shown in Figures $6 \mathrm{~b}$ and $6 \mathrm{c}$. These values are much higher than the value of yield stress. Therefore, the average value of shearing force, $\tau d A$, on cracked section is higher than the assumption of NSC method.

For the case of deep crack, $a / t \geq 0.75$, although there is stress singularity ahead the crack front, the value of average shearing force is still lower than the NSC assumption. This is because, when the cross section area is greatly reduced due to crack, the values of stresses acting on the area opposite to the crack location must decrease in order to maintain a self-equilibrated distribution of stress, as shown in Figures 6(d) and 6(e). According to this reason, the limit torsion moment obtained from the FE-analysis is lower than the one predicted from Eq.(3).

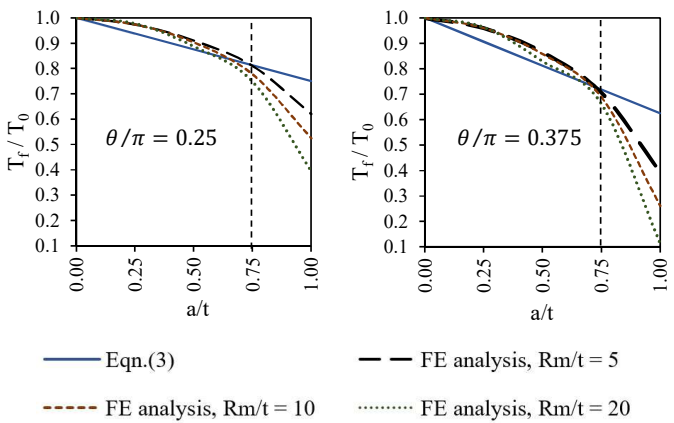

Fig. 5. Comparison of $T_{f} / T_{0}$ versus $\theta / \pi$ between NSC approach and $\mathrm{FE}$ analysis. 


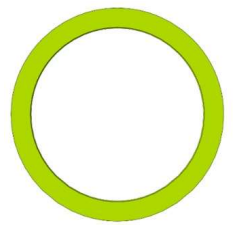

(a)

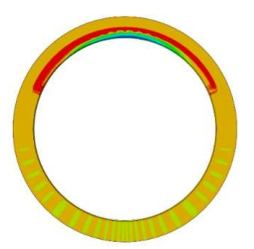

(c)

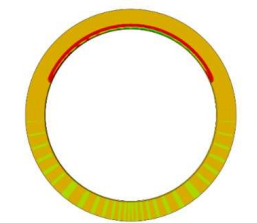

(b)

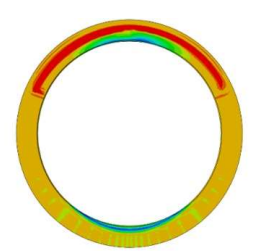

(d)
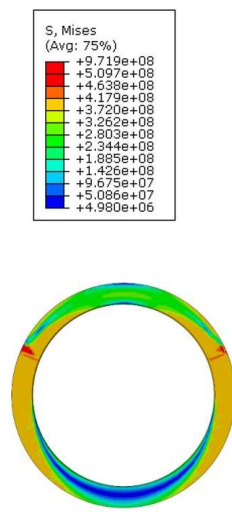

(e)
Fig. 6. The contour plot of von Mises stress at yielding state on the crack plane for the case of $\theta / \pi=0.375$ :

(a) $a / t=0.25$, (b) $a / t=0.50$, (c) $a / t=0.75$ and (d) $a / t=1.00$.

\subsection{The possible candidate for plastic collapse torsion solution}

According to section 4.2, for the case of $a / t<0.75$, the value of limit moment estimated by NSC approach, Eq.(3), is slightly lower than one obtained from FEanalysis. As, the difference is not over 5\%, Eq.(3) can be used to obtain the conservative solution of the plastic collapse torsional moment.

However, for the case of deeper crack with $a / t \geq$ 0.75 , the limit load obtained from FE-analysis is much lower than the results from Eq.(3). Therefore, the estimation of collapse torsional moment by Eq.(3) may not be safe. According to this, the following linear approximation based on the FE analysis is proposed.

$$
\begin{aligned}
\frac{T}{T_{0}}= & 0.5817+0.0258\left(\frac{R_{m}}{t}\right)+3.1165\left(\frac{\theta}{\pi}\right) \\
& +0.5468\left(\frac{a}{t}\right)-0.0218\left(\frac{R_{m}}{t}\right)\left(\frac{\theta}{\pi}\right) \\
& -4.9732\left(\frac{\theta}{\pi}\right)\left(\frac{a}{t}\right)-0.0331\left(\frac{a}{t}\right)\left(\frac{R_{m}}{t}\right)
\end{aligned}
$$

Eq.(6) provides the values of $T_{f} / T_{0}$ within $10 \%$ of $\mathrm{FE}$ results at $a / t \geq 0.75,2 \theta \leq 135^{\circ}$ and $5 \leq R_{m} / t \leq 20$.

\section{Conclusion}

In this work, both the NSC approach and 3D FE model are used to analyze plastic collapse load for cylinder with circumferential crack subjected to torsional moment. The material considered in this work is assumed to be elasticperfectly plastic. The influence of geometric parameters of crack and cylinder, such as $R_{m} / t, a / t$ and $\theta / \pi$ on the solution of plastic collapse load are investigated. The following conclusions can be made :

1. As material behaves elastically, the change of crack ligament area does not affect the slope of the relationship between torsion moment and torsion angle.
2. The ratio $R_{m} / t$ affects slightly on the change of $T_{f} / T_{0}$ when value of $a / t$ is less than 0.75 . However, when the values of $a / t$ is higher, the value of limit torsion moment changes significantly according to the value of $R_{m} / t$.

3. For the case of $a / t<0.75$, the values of limit torsion moment can be estimated by NSC analysis, Eq.(3). However, for the case of deeper crack, $a / t \geq 0.75$, the limit load solution predicted by NSC approach may not be safe as it provides very high value of $T_{f} / T_{0}$, comparing with $\mathrm{FE}$ analysis.

4. For the case of deeper crack with $a / t \geq 0.75$, the limit torsion moment solution can be estimated by linear approximation in Eq.(6), with the difference not over than $10 \%$ from FE analysis.

\section{References}

1. S.Rahmana and G.Wilkowskib, "Net-section-collapse analysis of circumferentially cracked cylinders-part I: arbitrary-shaped cracks and generalized equations," Engineering Fracture Mechanics, vol.61, pp.191-211, 1998.

2. Y.J.Kim, D.J.Shim, N.Kamran, Y.J.Kim, S.S.Hwang and J.S.Kim, "Finite element based plastic limit loads for cylinders with part-through surface cracks under combined loading," International Journal of Pressure Vessels and Piping, vol.80, pp.527-540, 2003.

3. Y.Lei and P.J.Budden, "Limit load solutions for thinwalled cylinders with circumferential cracks under combined internal pressure, axial tension and bending," The Journal of Strain Analysis for Engineering Design, vol.39(6), pp.673-683, 2004.

4. Y.Lei, Y.Li and Z.Gao, "Global limit load solutions for thick-walled cylinders with circumferential cracks under combined internal pressure, axial force and bending moment Part I: Theoretical solutions," International Journal of Pressure Vessels and Piping, vol.114-115, pp.23-40, 2014.

5. Y.Li, K.Hasegawa, P.H.Hoang and B.Bezensek, "Prediction Method for Plastic Collapse of Circumferentially Cracked Pipes Subjected to Combined Bending and Torsion Moments," 2012 ASME Pressure Vessels and Piping Division Conference, Toronto, Ontario, Canada, July 15-19, 2012.

6. K.Hasegawa, Y.Li and K.Osakabe, "Collapse loads for circumferentially through-wall cracked pipes subjected to combined torsion and bending moments," Engineering Fracture Mechanics 123 (2014) 77-85.

7. A.C.Ugural and S.K.Fenster, "Advanced Strength and Applied Elasticity," 3rd ed, PTR Prentice Hall, Englewood Cliffs, New Jersey

8. Hibbitt, Karlsson and Sorensen Inc, "ABAQUS/Standard User Manual," Rhode Island.

9. Y.Li, Y.Lei and Z.Gao, "Global limit load solutions for thick-walled cylinders with circumferential cracks under combined internal pressure, axial force and bending moment _ Part II: Finite element validation," International Journal of Pressure Vessels and Piping, vol.114-115, pp.41-60, 2014. 